\title{
SCF Cycle Convergence, Structure Optimization, and Vibrational Modes of Coumarin ( $\alpha$-Benzopyrone)
}

\author{
G. Babaji* \\ Department of Physics, Bayero University, Kano, Nigeria. \\ * Corresponding author. Tel.+2348036035096; email: gbabaji.phy@buk.edu.ng \\ Manuscript submitted March 6, 2015; accepted June 4, 2015. \\ doi: 10.17706/ijapm.2015.5.3.206-217
}

\begin{abstract}
Coumarin is a natural compound found in many plants. It has been successfully employed in dye lasers, and since 1996 it's potentiality in the development of a high efficiency dye sensitized solar cell is being investigated. In this work, the Fritz-Haber Institut ab initio Molecular Dynamic Simulation code, FHI-aims was used to investigate the convergence of the Self-Consistency Field Cycle, obtain the most stable ground state atomic coordinates, and to generate and analyze the vibrational modes of coumarin ( $\alpha$-Benzopyrone. The exchange-correlation interactions are treated by the Linear Density Approximation. The ground state total energy of the molecule was found to be $-13531.52 \mathrm{eV}$. The result of the vibrational modes analysis confirmed that the optimized geometry is indeed a local minimum and not a saddle point. A total of 51 vibrational modes are identified with highest IR absorption of $14.15 \mathrm{D}^{2} \AA^{-2}$ at $1789.57 \mathrm{~cm}^{-1}$. An important finding of the Self-Consistency Field Cycle convergence shows an error in the FHI-aims user manual.
\end{abstract}

Key words: Coumarins, density functional theory, molecular dynamics.

\section{Introduction}

The Benzopyrones are a group of compounds whose members include coumarins and flavornoids. The basic structures of the coumarins and flavornoids are given in Fig. 1. Coumarins owe their class name to 'Coumarou', the vernacular name of the tonka bean (Dipteryx odorata Willd., Fabaceae), from which coumarin itself was isolated in 1820 [1].

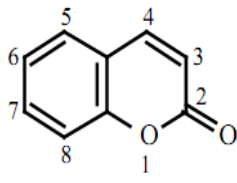

[A] $\alpha$-Benzopyrone

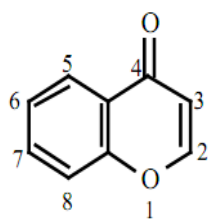

[B] $\gamma$-Benzopyrone

Fig. 1. The chemical structures of benzopyrone subclasses, with the basic coumarin structure (benzo $\alpha$-pyrone) [A], and flavonoid (benzo- $\gamma$-pyrone) structure [B].

There are four main coumarin sub-types: the simple coumarins, furanocoumarins, pyranocoumarins and the pyrone-substituted coumarins. Coumarins comprise a very large class of compounds found throughout the plant kingdom. They are found at high levels in some essential oils, particularly cinnamon bark oil 
(7,000 ppm), cassia leaf oil (up to 87,300 ppm) and lavender oil. Coumarin is also found in fruits (e.g. bilberry, cloudberry), green tea and other foods such as chicory. Most coumarins occur in higher plants, with the richest sources being the Rutaceae and Umbelliferae. Although distributed throughout all parts of the plant, the coumarins occur at the highest levels in the fruits, followed by the roots, stems and leaves [2].

Coumarins constitute an important class of naturally occurring compounds; they are widely used in the perfume, cosmetic, agrochemical and pharmaceutical industries. Several coumarin derivatives have been synthetically prepared and reported to possess cardiovascular, ageing, antibacterial and photosensitive properties. The chemistry of coumarins has received much attention and is used by chemists to develop several useful products [3]. Coumarins are also used as sensitizers in Dye Sensitized Solar Cells (DSSC).

Photovoltaic devices are based on the concept of charge separation at an interface of two materials of different conduction mechanism. To date this field has been dominated by solid-state junction devices, usually made of silicon. The dominance of the photovoltaic field by inorganic solid-state junction devices is now being challenged by the emergence of a third generation of cells, based, for example, on nanocrystalline and conducting polymers films. These offer the prospective of very low fabrication cost and present attractive features that facilitate market entry. It is now possible to depart completely from the classical solid state junction device, by replacing the contacting phase to the semiconductor by an electrolyte, liquid, gel or solid, thereby forming a photo-electrochemical cell. The phenomenal progress realized recently in the fabrication and characterization of nanocrystalline materials has opened up vast new opportunities for these systems. Contrary to expectation, devices based on interpenetrating networks of mesoscopic semiconductors have shown strikingly high conversion efficiencies, which compete with those of conventional devices. The prototype of this family of devices is the dye-sensitzed solar cell, which realizes the optical absorption and the charge separation processes by the association of a sensitizer as light-absorbing material with a wide band gap semiconductor of nanaocrystalline morphology [4].

The history of dye sensitized solar cells (DSSC) started in 1972 with a chlorophyll sensitized zinc oxide (ZnO) electrode. For the first time, photons were converted into an electric current by charge injection of excited dye molecules into a wide bandgap semiconductor [5]. Since 1991, f ollowing the demonstration of dye sensitized solar cells (DSSCs) for the first time by Prof Michael Gratzel at EPFL,[6] DSSCs have been attracting attention of both researchers and industries worldwide. Due to its low material cost, easy and inexpensive methods of fabrication and reasonably good power conversion efficiency, [7], [8] DSSCs are being considered to be a potential alternative to expensive conventional inorganic solar cells. Unlike silicon solar cells, electrons and holes in a DSSC are transported in two different phases, $\mathrm{TiO}_{2}$ and electrolyte respectively, and because of which the chances of recombination in the cell become low. Hence, DSSCs do not require ultra high pure materials unlike inorganic solar cells. In addition, DSSCs have been proved to perform better than conventional solar cells, without significant change in the power conversion efficiency, in diffused light [7], [9] and at moderate temperature - up to $50^{\circ} \mathrm{C}$ [10]. Moreover, these cells can be made on flexible substrates [11], [12] and thus find applications on curved surf aces like clothes, bags, car tops, etc. Transparency of these cells and their invariant performance under the diffused light make these cells more attractive for indoor applications and as panels on windows and doors.

Density-functional theory is one of the most popular and successful quantum mechanical approaches to matter. It is nowadays routinely applied for calculating, e.g., the binding energy of molecules in chemistry and the band structure of solids in physics. First applications relevant for fields traditionally considered more distant from quantum mechanics, such as biology and mineralogy are beginning to appear. Superconductivity, atoms in the focus of strong laser pulses, relativistic effects in heavy elements and in atomic nuclei, classical liquids, and magnetic properties of alloys have all been studied with DFT. DFT owes this versatility to the generality of its fundamental concepts and the flexibility one has in implementing 
them. In spite of this flexibility and generality, DFT is based on quite a rigid conceptual framework.

The most prominent modern DSSCs, the so-called Gratzel cells, consist of porous layers of titanium dioxide nanoparticles covered by molecular dyes that absorb sun light. While most efficient sensitizers are composed of ruthenium dye complexes, intense research is conducted so as to find molecular alternatives which are cheaper, easier to synthesize, and free from the resource limitations related to the noble metal ruthenium. As a promising direction, Hara and coworkers [13] demonstrated that coumarin-based dyes, such as the so-called NKX-2xxx family, could lead to conversion efficiencies approaching that of ruthenium-based DSSCs. Also, recently Faber et al. [14] studied within the many-body Green's function GW and Bethe-Salpeter formalisms the excitation energies of several coumarin dyes proposed as an efficient alternative to ruthenium complexes for dye-sensitized solar cells. Due to their internal donor-acceptor structure, these chromophores present low-lying excitations showing a strong intramolecular charge-transfer character.

The ongoing search for an alternative to the organometallic complex sensitizers is what motivated this work. The DFT based code, fhi-aims is used in performing the molecular relaxations and vibrations. An important objective of the work is to develop a systematic procedure to be adopted in molecular geometry optimization using fhi-aims.

\section{Theoretical Background}

\subsection{Density Functional Theory}

Let us consider a system of $N$ interacting (spinless) electrons under an external potential $V(\mathbf{r})$ (usually the Coulomb potential of the nuclei). If the system has a nondegenerate ground state, it is obvious that there is only one ground-state charge density $n(\mathbf{r})$ that corresponds to a given $V(\mathbf{r})$. In 1964 Hohenberg and Kohn [15] demonstrated the opposite, i.e. there is only one external potential $V(\mathbf{r})$ that yields a given ground-state charge density $n(\mathbf{r})$, and in $1965 \mathrm{Kohn}$ and Sham[16] reformulated the problem in a more familiar form and opened the to practical applications of DFT. Let us consider a many-electron Hamiltonian $H=T+U+V$, with ground state wavefunction $\Psi$, where $T$ is the kinetic energy, $U$ the electron-electron interaction, and $V$ the external potential. The charge density $n(\mathbf{r})$ is defined as

$$
n(\mathbf{r})=N \int\left|\Psi\left(\mathbf{r}, \mathbf{r}_{2}, \mathbf{r}_{3}, \mathbf{r}_{4}, \ldots \mathbf{r}_{N}\right)\right|^{2} d \mathbf{r}_{2} \ldots d \mathbf{r}_{N}
$$

A straightforward consequence of the first Hohenberg and Kohn theorem is that the ground state energy, $E$ is also uniquely determined by the ground-state charge density. In mathematical terms $E$ is a functional $E[n(\mathbf{r})]$ of $n(\mathbf{r})$. We can write

$$
E[n(\mathbf{r})]=\langle\Psi|T+U+V| \Psi\rangle=\langle\Psi|T+U| \Psi\rangle+\langle\Psi|V| \Psi\rangle=F[n(\mathbf{r})]+\int n(\mathbf{r}) V(\mathbf{r}) d \mathbf{r}
$$

where $F[n(r)]$ is a universal functional of the charge density $n(\mathbf{r})$ (and not of $V(\mathbf{r})$ ). For this functional a variational principle holds: the ground-state energy is minimized by the ground-state charge density. In this way, DFT exactly reduces the N-body problem to the determination of a 3-dimensional function $n(\mathbf{r})$ which minimizes a functional $E[n(\mathbf{r})]$. Unfortunately this is of little use as $F[n(\mathbf{r})]$ is not known.

It is convenient to rewrite the energy functional as follows:

$$
E=T_{S}[n(\mathbf{r})]+E_{H}[n(\mathbf{r})]+E_{x c}[n(\mathbf{r})]+\int n(\mathbf{r}) V(\mathbf{r}) d \mathbf{r} .
$$

The first term is the kinetic energy of the non-interacting electrons: 


$$
T_{S}[n(\mathbf{r})]=-\frac{\hbar^{2}}{2 m} 2 \sum_{i} \int \psi_{i}^{*}(\mathbf{r}) \nabla^{2} \psi_{i}(\mathbf{r}) d \mathbf{r}
$$

The second term, called the Hartree energy contains the electrostatic interactions between clouds of charge:

$$
E_{H}[n(\mathbf{r})]=\frac{e^{2}}{2} \int \frac{n(\mathbf{r}) n\left(\mathbf{r}^{\prime}\right)}{\left|\mathbf{r}-\mathbf{r}^{\prime}\right|} d \mathbf{r} d \mathbf{r}^{\prime}
$$

The third term, called the exchange-correlation energy, contains all the remaining terms.

\subsection{Linear Density Approximation (LDA)}

There are many formalism put forward to approximate exchange-correlation interactions, the LDA been one of them. Also, several excellent reviews have made e.g. [17], [18]. A brief introduction to LDA is given below.

The local density approximation (LDA) is the basis of all approximate exchange-correlation functionals. At the centre of this model is the idea of a uniform electron gas. This is a system in which electrons move on a positive background charge distribution such that the total ensemble is neutral.

The central idea of LDA is the assumption that we can write Exc in the following form

$$
E_{X C}^{L D A}(\rho)=\int \rho(\mathbf{r}) \varepsilon_{X C}(\rho(\mathbf{r})) d \mathbf{r}
$$

Here, $\varepsilon_{X C}(\rho(\mathbf{r}))$ is the exchange-correlation energy per particle of a uniform electron gas of density $\rho(\mathbf{r})$. This energy per particle is weighted with the probability $\rho(\mathbf{r})$ that there is an electron at this position. The quantity $\varepsilon_{X C}(\rho(\mathbf{r}))$ can be further split into exchange and correlation contributions,

$$
\varepsilon_{X C}(\rho(\mathbf{r}))=\varepsilon_{X}(\rho(\mathbf{r}))+\varepsilon_{C}(\rho(\mathbf{r}))
$$

The accuracy of the LDA for the exchange energy is typically within $10 \%$, while the normally much smaller correlation energy is generally over estimated by up to a factor 2 . The two errors typically cancle partially. Experience has shown that the LDA gives ionization energies of atoms, dissociation energies of molecules and cohesive energies with a fair accuracy of typically 10-20\%. However, the LDA gives bond lengths of molecules and solids typically with an astonishing accuracy of about $2 \%$. This moderate accuracy that LDA delivers is certainly insufficient for most applications in Chemistry. LDA can also fail in systems, like heavy fermions, so dominated by electron-electron interaction effects.

\section{FHI-Aims}

FHI-aims ("Fritz Haber Institute ab initio molecular simulations") is a computer program package for computational materials science based only on quantum-mechanical first principles. The main production method is density functional theory to compute the total energy and derived quantities of molecular or solid condensed matter in its electronic ground state. In addition, FHI-aims allows to describe electronic single quasi particle excitations in molecules using different self-energy formalisms (GW and MP2), and wave-function based molecular total energy calculation based on Hartree-Fock and many-body perturbation theory (MP2, RPA, SOSEX, or the more encompassing renormalized second-order perturbation theory, RPT2) [19]. 
FHI-aims requires exactly two input files-control.in and geometry.in-located in the same directory from which the FHI-aims binary is invoked. To start FHI-aims, no further input should be needed.

Geometry.in contains only information directly related to the atomic structure for a given calculation. This obviously includes atomic positions, with a description of the particulars of each element (or species) expected in control.in. In addition, lattice vectors may be defined if a periodic calculation is required. Any other information is only given here if it is directly tied to the atom in question, such as an initial charge, initial spin moment, relaxation constraint etc. The order of lines is irrelevant, except that information specific to a given atom must follow after the line specifying that atom, and before any following atom is specified.

control.in contains all other runtime-specific information. Typically, this file consists of a general part, where, again, the particular order of lines is unimportant. In addition, this file contains species subtags that are references by geometry.in. Within the description of a given species, the order of lines is again unimportant, but all information concerning the same species must follow the initial species tag in one block.

\section{Result and Discussion}

\subsection{Coordinates and Energy of the Starting Molecule}

The atomic coordinates and energy components of the starting molecule i.e the one built and 'clean' by using the ArgusLab code are shown in Table 1 and Table 2 respectively.

Table 1. Atomic Coordinates of the $\alpha$-Benzopyrone Molecule Built and Clean in Argus

\begin{tabular}{|c|l|l|l|l|}
\hline $\mathrm{S} / \mathrm{N}$ & ATOM & $X$-Coordinate $(\AA)$ & $Y$-Coordinate $(\AA)$ & $Z$-Coordinate $(\AA)$ \\
\hline 1 & $\mathrm{C}$ & -2.87148961 & 1.34583554 & -0.10819989 \\
\hline 2 & $\mathrm{C}$ & -1.62829921 & 0.70689478 & -0.13061228 \\
\hline 3 & $\mathrm{C}$ & -1.57731403 & -0.77140857 & -0.09928074 \\
\hline 4 & $\mathrm{C}$ & -4.05579823 & 0.60457420 & -0.00770509 \\
\hline 5 & $\mathrm{C}$ & -4.04358866 & -0.79438551 & 0.06801124 \\
\hline 6 & $\mathrm{C}$ & -2.84381119 & -1.50600336 & 0.03078531 \\
\hline 7 & $\mathrm{C}$ & 0.81886652 & 0.64111194 & 0.29287633 \\
\hline 8 & $\mathrm{C}$ & 0.78671032 & -0.74358499 & -0.35863033 \\
\hline 9 & $\mathrm{O}$ & 1.84026436 & -1.56195665 & 0.17464496 \\
\hline 10 & $\mathrm{O}$ & -0.47174618 & -1.39206393 & -0.15852364 \\
\hline 11 & $\mathrm{C}$ & -0.34612344 & 1.48185492 & -0.22640806 \\
\hline 12 & $\mathrm{H}$ & -2.92657479 & 2.42944845 & -0.15739949 \\
\hline 13 & $\mathrm{H}$ & -5.00691956 & 1.12745384 & 0.01703092 \\
\hline 14 & $\mathrm{H}$ & -4.98201322 & -1.33332225 & 0.15353699 \\
\hline 15 & $\mathrm{H}$ & -2.84869607 & -2.59036396 & 0.08402431 \\
\hline 16 & $\mathrm{H}$ & -0.41107143 & 2.42458699 & 0.36376029 \\
\hline 17 & $\mathrm{H}$ & 1.78174352 & 1.15029887 & 0.06208919 \\
\hline
\end{tabular}

Table 2. Energy Components of the Benzopyrone Molecule Built and Clean in Argus

\begin{tabular}{ccc} 
Component & Energy & \\
& MM Bond & 0.00234700 \\
MM Angle & 0.00196832 & \\
MM Dihedral & 0.00338000 & \\
MM ImpTor & 0.00001930 & \\
MM vdW & 0.02112998 & \\
\hdashline Total & 0.02884460 a.u. & \\
$18.10027440 \mathrm{kcal} / \mathrm{mol}$
\end{tabular}

\subsection{Self-Consistency Field Cycle (SCFC) Convergence}


A default control.in (having the minimum basis size) was used to relax the initial molecule in order to investigate the effect of some important Fhi-aims keywords. The variation of total energy as the molecule is relaxed is shown in Fig. 2, while Table 3 summarizes the result of the relaxation process. Table 4, Table 5, and Table 6 show respectively the effect of the parameters sc_accuracy_etot, sc_accuracy_eev, and sc_accuracy_rho. The effect of generating, and using a restart file in the relaxation is shown in Table 7.

Table 3. Summary of the Result of Relaxing the Initial Molecule

\begin{tabular}{|l|l|}
\hline Total Energy, E (eV) & -13389.16239582 \\
\hline Nunber of Self-consistency cycles & 205 \\
\hline Number of Relaxation Steps & 19 \\
\hline Total Time (s) & 678.418 \\
\hline
\end{tabular}

Table 4. Effect of Parameter sc_accuracy_etot

\begin{tabular}{|c|c|c|c|c|}
\hline Value of etot & Total Energy (eV) & No. SCFC & $\begin{array}{c}\text { No. Relx. } \\
\text { Steps }\end{array}$ & $\begin{array}{c}\text { Time } \\
(\mathrm{s})\end{array}$ \\
\hline not used & -13389.16329 & 205 & 19 & 681.53 \\
\hline 0 & SCF cycle not converged & 1000 & 0 & 2212.1 \\
\hline 1.00E-006 & $\mathbf{- 1 3 3 8 9 . 1 6 3 2 9}$ & $\mathbf{2 0 5}$ & $\mathbf{1 9}$ & $\mathbf{6 7 7 . 6 1}$ \\
\hline $1.00 \mathrm{E}-005$ & -13389.16329 & 205 & 19 & 680.2 \\
\hline $1.00 \mathrm{E}-003$ & -13389.16329 & 205 & 19 & 680.3 \\
\hline $1.00 \mathrm{E}-001$ & -13389.16329 & 205 & 19 & 681.47 \\
\hline
\end{tabular}

Table 5. Effect of Parameter sc_accuracy_eev

\begin{tabular}{|c|c|c|c|c|}
\hline Value of eev & Total Energy (eV) & No. SCFC & $\begin{array}{c}\text { No. Relx. } \\
\text { Steps }\end{array}$ & $\begin{array}{c}\text { Time } \\
(\mathrm{s})\end{array}$ \\
\hline not used & -13389.16329 & 205 & 19 & 678.95 \\
\hline 0 & SCF cycle not converged & 1000 & 0 & 2247.7 \\
\hline 0.01 & -13389.16329 & 205 & 19 & 677.61 \\
\hline $\mathbf{0 . 0 5}$ & $\mathbf{- 1 3 3 8 9 . 1 6 3 2 9}$ & $\mathbf{2 0 5}$ & $\mathbf{1 9}$ & $\mathbf{6 7 6 . 7 8}$ \\
\hline 0.1 & -13389.16329 & 205 & 19 & 679.01 \\
\hline 0.5 & -13389.16329 & 205 & 19 & 677.19 \\
\hline
\end{tabular}

Table 6. Effect of Parameter $s c_{-} a c c u r a c y \_r h o$

\begin{tabular}{|c|c|c|c|c|}
\hline Value of $r h o$ & Total Energy (eV) & No. SCFC & $\begin{array}{c}\text { No. Relx. } \\
\text { Steps }\end{array}$ & $\begin{array}{c}\text { Time } \\
(\mathrm{s})\end{array}$ \\
\hline not used & -13389.16329 & 205 & 19 & 707.31 \\
\hline 0 & SCF cycle not converged & 1000 & 0 & 2252.1 \\
\hline $\mathbf{1 . 0 0 E - 0 0 4}$ & $\mathbf{- 1 3 3 8 9 . 1 6 3 2 9}$ & $\mathbf{2 0 5}$ & $\mathbf{1 9}$ & $\mathbf{6 7 7 . 6 1}$ \\
\hline 1.00E-003 & -13389.16329 & 205 & 19 & 716.35 \\
\hline 1.00E-002 & -13389.16329 & 205 & 19 & 706.31 \\
\hline 1.00E-001 & -13389.16329 & 205 & 19 & 707.46 \\
\hline
\end{tabular}

Table 7. Effect of Restart File

\begin{tabular}{|c|c|c|c|c|}
\hline TYPE OF RUN & Total Energy $(\mathrm{eV})$ & $\begin{array}{c}\text { No. } \\
\text { SCFC }\end{array}$ & $\begin{array}{c}\text { No. } \\
\text { Relx. } \\
\text { Steps }\end{array}$ & Time (s) \\
\hline without restart file & -13389.16329 & 205 & 19 & 677.61 \\
\hline generating & -13389.16329 & 205 & 19 & 679.22 \\
\hline using & -13389.16329 & 205 & 19 & 674.79 \\
\hline
\end{tabular}

\subsection{Effect of Basis Size}


The effect of the size of the basis (i.e. the number of basis functions used in expanding the wave function) is investigated by relaxing the initial molecule using the default control.in file. This was done without employing a restart file. The result is given in Table 8. It should be noted from Table 8 that it took about two hours for the relaxation to be completed with tier 3 basis.

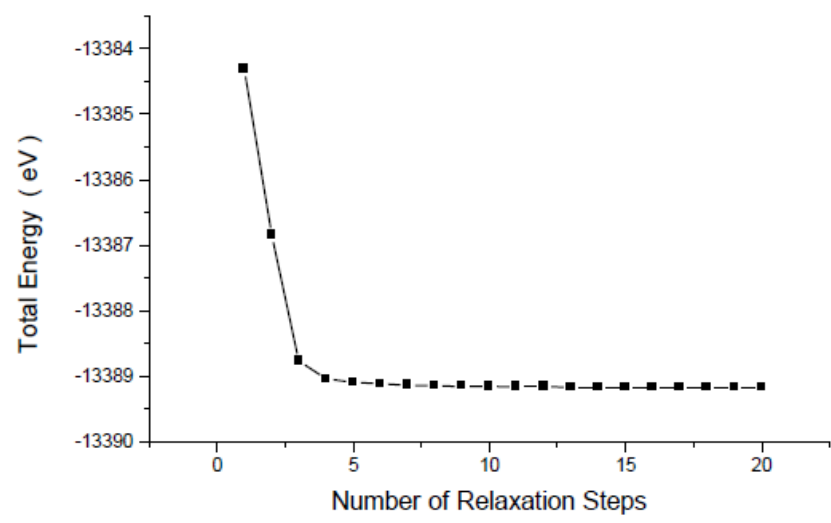

Fig. 2. Variation of total energy with number of relazation steps.

Table 8. Summary of the Result of Relaxing the Initial Molecule with Different Basis Size

\begin{tabular}{|c|c|c|c|c|}
\hline Basis size & Total Energy (eV) & $\begin{array}{c}\text { No. } \\
\text { SCFC }\end{array}$ & $\begin{array}{c}\text { No. } \\
\text { Relx. } \\
\text { Steps }\end{array}$ & Time (s) \\
\hline Minimum & -13389.16329 & 205 & 19 & 677.61 \\
\hline teir1 & -13415.37466 & 230 & 21 & 1261.8 \\
\hline teir2 & -13416.26015 & 233 & 21 & 3772.5 \\
\hline teir3 & -13416.30460 & 236 & 21 & 6965.5 \\
\hline
\end{tabular}

\subsection{Geometry Optimization}

A geometry optimization using the default control.in file was done by using an improved atomic coordinates as the basis size is increased. For example, the geometry of the initial molecule was optimized employing the minimum basis and the optimized geometry was used as the starting geometry when the basis is changed to tier 1. Similarly, the output geometry using tier 1 was used as the starting geometry for the tier 2 basis, and so on. A restart file was not used because the time taken to perform geometry optimization with this stratergy is small. The summary of the result is given in Table 9.

Table 9. Summary of Geometry Optimization Result

\begin{tabular}{|c|c|c|c|c|c|}
\hline & No. of Basis Functions & & $\begin{array}{c}\text { No. } \\
\text { BCFC }\end{array}$ & $\begin{array}{c}\text { Relx. } \\
\text { Steps }\end{array}$ & Time (s) \\
\hline Minimum & 10 & -13415.37466 & 205 & 19 & 677.61 \\
\hline teir1 & 18 & -13416.26018 & 180 & 10 & 639.6 \\
\hline teir2 & 32 & -13416.30465 & 49 & 4 & 796.52 \\
\hline teir3 & 44 & -13416.32412 & 23 & 1 & 723.41 \\
\hline teir4 & 54 & -13416.31448 & 69 & 6 & 3514.4 \\
\hline Maximum & 61 & -13416.31448 & 16 & 0 & 908.63 \\
\hline
\end{tabular}

\subsection{Optimizing Key Parameters in Control.in File}

A relaxation run for the purpose of generating a restart file was done using the default control.in file and 
the final geometry of 4.4 (i.e. maximum basis). Some important parameters in control.in were optimized by relaxing the 'final geometry' several times. The results are given in Table 10, Table 11, Table 12, and Table 13 , where the entry for an optimum parameter is shown bolded.

Table 10. Result of Optimizing the Parameter default_initial_moment

\begin{tabular}{|c|c|c|c|c|}
\hline Tolerance & Total Energy (eV) & $\begin{array}{c}\text { No. } \\
\text { SCFC }\end{array}$ & $\begin{array}{c}\text { No. Relx. } \\
\text { Steps }\end{array}$ & Time (s) \\
\hline 0.2 & -13416.3144833403 & 16 & 0 & 926.798 \\
\hline 0.15 & -13416.3144833187 & 16 & 0 & 893.612 \\
\hline 0.1 & -13416.3144833006 & 16 & 0 & 908.629 \\
\hline 0.05 & -13416.3144832852 & 16 & 0 & 909.049 \\
\hline 0.5 & -13416.3144833361 & 16 & 0 & 896.06 \\
\hline 0.02 & -13416.3144832844 & 15 & 0 & 845.821 \\
\hline 1 & -13416.3144833256 & 17 & 0 & 939.967 \\
\hline 0.01 & -13416.3144833197 & 15 & 0 & 841.581 \\
\hline 2 & Hazardous setting - please correct. Default initial moment per & & & \\
\hline $5.00 \mathrm{E}-003$ & atom is larger than number of electrons on species H. & 15 & 0 & 841.765 \\
\hline 1.00E-003 & -13416.3144833395 & 15 & $\mathbf{0}$ & $\mathbf{8 4 1 . 2 3 3}$ \\
\hline $1.00 \mathrm{E}-004$ & -13416.3144833484 & 15 & 0 & 846.513 \\
\hline $1.00 \mathrm{E}-005$ & -13416.3144833484 & 15 & 0 & 846.917 \\
\hline $1.00 \mathrm{E}-006$ & -13416.3144833484 & 15 & 0 & 847.645 \\
\hline $1.00 \mathrm{E}-007$ & -13416.3144833484 & 15 & 0 & 847.549 \\
\hline
\end{tabular}

Table 11. Result of Optimizing the Parameter gaussian Width

\begin{tabular}{|r|c|r|r|r|}
\hline width () & Total Energy (eV) & No. SCFC & No. Relx. Steps & Time (s) \\
\hline 0.01 & -13416.3144834079 & 15 & 0 & 833.42 \\
\hline 0.001 & -13416.3144834079 & 15 & 0 & 833.072 \\
\hline $\mathbf{0 . 1}$ & $\mathbf{- 1 3 4 1 6 . 3 1 4 4 8 3 4 0 7 9}$ & $\mathbf{1 5}$ & $\mathbf{0}$ & $\mathbf{8 3 0 . 7 5 6}$ \\
\hline
\end{tabular}

Table 12. Result of Optimizing the Parameter Charge_mix_param

\begin{tabular}{|r|c|c|c|c|}
\hline value & Total Energy (eV) & No. SCFC & No. Relx. Steps & Time (s) \\
\hline 1 & -13416.3144833123 & 17 & 0 & 939.643 \\
\hline 0.9 & -13416.3144833488 & 16 & 0 & 896.16 \\
\hline 0.8 & -13416.3144833411 & 15 & & 845.845 \\
\hline 0.7 & -13416.3144833339 & 15 & 0 & 832.736 \\
\hline 0.6 & -13416.3144833484 & 15 & 0 & 846.513 \\
\hline $\mathbf{0 . 5}$ & $\mathbf{- 1 3 4 1 6 . 3 1 4 4 8 3 4 0 7 9}$ & $\mathbf{1 5}$ & $\mathbf{0}$ & $\mathbf{8 3 3 . 4 2 0}$ \\
\hline 0.4 & -13416.3144833686 & 15 & 0 & 847.857 \\
\hline 0.3 & -13416.3144833205 & 16 & 0 & 880.815 \\
\hline 0.2 & -13416.3144833573 & 17 & 0 & 931.750 \\
\hline
\end{tabular}

Table 13. Result of Optimizing Parameter Methfessel-paxton occupation type

\begin{tabular}{|c|c|c|c|c|}
\hline Width & Total Energy (eV) & No. SCFC & No. Relx. Steps & Time (s) \\
\hline 0.01 & -13416.3144834079 & 15 & 0 & 834.972 \\
\hline 0.001 & -13416.3144834079 & 15 & 0 & 831.464 \\
\hline 0.1 & -13416.3144834079 & 15 & 0 & 830.180 \\
\hline
\end{tabular}

\subsection{Single-Point Calculations with LDA Exchane-Correlations Functions}


The results of 4.2 and 4.5 were used to set up an optimized controlin file which was the used to run several single-point calculations using different LDA exchange-correlation functional supported in FHI-aims. Table 14 shows the energies for the various functionals. The Rpbe functional provided the lowest energy, while Vwn took the shortest time.

Table 14. Total Energy for Different Types of LDA Exchange-Correlation Functions

\begin{tabular}{|l|l|l|l|}
\hline XC & Total Energy (eV) & No. SCFC & Time (s) \\
\hline Pw-lda & -13416.3144839089 & 13 & 717.537 \\
\hline Pz-lda & -13416.0917537102 & 13 & 716.697 \\
\hline Vwn & -13416.8811204728 & 13 & 716.453 \\
\hline am05 & -13431.1272600285 & 14 & 921.102 \\
\hline Blyp & -13526.8470187172 & 13 & 847.021 \\
\hline Pbe & -13514.9043703384 & 14 & 915.661 \\
\hline Pbeint & -13467.9966510341 & 14 & 918.835 \\
\hline Pbesol & -13461.7572763328 & 14 & 916.689 \\
\hline Rpbe & -13531.5152006310 & 14 & 968.641 \\
\hline Revpbe & -13527.1583189776 & 14 & 965.12 \\
\hline
\end{tabular}

\subsection{Vibrational Modes}

The exchange-correlation function was set to vwn and the FHI-aims vibrations run. The result is given in Table 15.

Table 15. Ground State Vibrational Modes, Frequencies, Zero point energies and IR-Intesities of $\alpha$-Benzopyrone

\begin{tabular}{|c|c|c|c|c|c|c|c|}
\hline $\begin{array}{l}\text { Mode } \\
\text { No. }\end{array}$ & Frequeny $\left(\mathrm{cm}^{-1}\right)$ & $\begin{array}{l}\text { Zero point } \\
\text { energy }(\mathrm{eV})\end{array}$ & $\begin{array}{c}\text { IR intensity } \\
\left(\mathrm{D}^{2} / \AA^{2}\right)\end{array}$ & $\begin{array}{l}\text { Mode } \\
\text { No. }\end{array}$ & Frequeny $\left(\mathrm{cm}^{-1}\right)$ & $\begin{array}{l}\text { Zero point } \\
\text { energy }(\mathrm{eV})\end{array}$ & $\begin{array}{c}\text { IR intensity } \\
\left(D^{2} / \AA^{2}\right)\end{array}$ \\
\hline 1 & -1.48967873 & -0.00009235 & 0.00000495 & 27 & 931.80643947 & 0.05776463 & 0.08431616 \\
\hline 2 & -0.44737202 & -0.00002773 & 0.00000051 & 28 & 967.95502170 & 0.06000556 & 0.00545537 \\
\hline 3 & -0.28441320 & -0.00001763 & 0.00000088 & 29 & 975.55890062 & 0.06047694 & 0.00771548 \\
\hline 4 & 7.43977048 & 0.00046121 & 0.03154877 & 30 & 1030.21705861 & 0.06386531 & 0.02348370 \\
\hline 5 & 8.84135659 & 0.00054809 & 0.03192527 & 31 & 1073.10129603 & 0.06652379 & 1.29418206 \\
\hline 6 & 12.14578643 & 0.00075294 & 0.03681390 & 32 & 1107.04128586 & 0.06862781 & 0.46198929 \\
\hline 7 & 97.85872687 & 0.00606647 & 0.03845171 & 33 & 1128.65376902 & 0.06996761 & 0.02841955 \\
\hline 8 & 160.02051185 & 0.00992001 & 0.09191590 & 34 & 1161.03617576 & 0.07197506 & 1.28902449 \\
\hline 9 & 255.78124327 & 0.01585641 & 0.01630871 & 35 & 1225.31086930 & 0.07595958 & 0.09343925 \\
\hline 10 & 301.42710243 & 0.01868610 & 0.02097602 & 36 & 1243.84462705 & 0.07710853 & 0.00115224 \\
\hline 11 & 371.16325837 & 0.02300919 & 0.00053302 & 37 & 1281.39160800 & 0.07943615 & 0.87941339 \\
\hline 12 & 444.82095809 & 0.02757538 & 0.03320170 & 38 & 1382.07212134 & 0.08567754 & 0.23171770 \\
\hline 13 & 451.22433792 & 0.02797234 & 0.15280670 & 39 & 1408.50347122 & 0.08731608 & 0.08477565 \\
\hline 14 & 484.54175696 & 0.03003776 & 0.07273632 & 40 & 1449.21229533 & 0.08983970 & 0.65576456 \\
\hline 15 & 524.27156419 & 0.03250069 & 0.14850473 & 41 & 1492.48933358 & 0.09252254 & 0.17738806 \\
\hline 16 & 547.93837213 & 0.03396785 & 0.01138579 & 42 & 1579.32859547 & 0.09790588 & 0.75128297 \\
\hline 17 & 608.24462525 & 0.03770636 & 0.15989382 & 43 & 1630.20746741 & 0.10105997 & 0.77784359 \\
\hline 18 & 680.79988498 & 0.04220421 & 0.04411763 & 44 & 1642.00070723 & 0.10179106 & 1.92844106 \\
\hline 19 & 731.47940797 & 0.04534594 & 0.03892890 & 45 & 1789.57834035 & 0.11093971 & 14.1523306 \\
\hline 20 & 745.95138874 & 0.04624309 & 1.28406310 & 46 & 3069.43738288 & 0.19028084 & 0.10926111 \\
\hline 21 & 765.12087919 & 0.04743144 & 0.07033064 & 47 & 3078.84383686 & 0.19086397 & 0.03783808 \\
\hline 22 & 768.05436092 & 0.04761330 & 0.00242664 & 48 & 3092.26968406 & 0.19169627 & 0.04794199 \\
\hline 23 & 821.36393428 & 0.05091807 & 1.15696083 & 49 & 3107.14779075 & 0.19261859 & 0.04710974 \\
\hline 24 & 861.30043977 & 0.05339382 & 0.06439713 & 50 & 3112.92682264 & 0.19297685 & 0.02761642 \\
\hline 25 & 876.76499281 & 0.05435250 & 0.58183084 & 51 & 3123.32378846 & 0.19362138 & 0.02073624 \\
\hline 26 & 920.21846245 & 0.05704627 & 0.72488106 & & & & \\
\hline
\end{tabular}




\subsection{Discussion}

In all self consistent schemes, it is necessary to have a stopping criteria. In this work, three of the FHI-aims keywords that determine the condition for terminating the self-consistency field cycle iterations namely sc_accuracy_etot, sc_accuracy_eev, and sc_accuracy_rho are investigated. The value of keyword sc_accuracy_eev, is a small positive real number [in eV], against which the difference of the eigenvalue sum between the present and previous s.c.f. iteration is checked. The value of keyword sc_accuracy_etot is a small positive real number [in eV], against which the difference of the total energy between the present and previous s.c.f. iteration is checked, while the value of keyword sc_accuracy_rho is a small positive real number [in electrons], against which the volumeintegrated root-mean square change of the charge density between the present and previous s.c.f. iteration is checked.

In the manuals (past and current) of FHI-aims it is stated that if the value of sc_accuracy-rho 'is set to zero or not given, the charge density will not be used as a convergence criterion'. Similar remarks are made on the values of . sc_accuracy_etot, and sc_accuracy_eev. However, the result given in Table 3 shows that there is big difference between setting any of these keywords to zero and not using them as a convergence criteria. If the value of any of these ( and similar keywords e.g. $s_{-}$accuracy_forces) is set to zero the SCFC will never converge but if any or all of them are not use at all, the SCFC wii converge normally.

Also the keyword restart has been studied in this work. Its usage takes the form; 'restart file' where file is a string, corresponding to the desired restart filename. The restart keyword saves and reads the final wave function of each scf-cycle to/or from file. If file is not yet present, the calculation simply writes that file during the run. If file is already present, it is read and the wave function contained therein is used to restart the calculation, instead of a fresh superposition of free atoms initialization. Normally, the use of the restart keyword shortens the time to make a calculation. However, it is seen in this work that the restart keyword need not be used if the starting geometry is a good one, the number of basis function is very small, and/or the cluster/molecule is small. A run in which the restart file is generated takes a longer period to complete.

The size of the basis is an important factor which determines the accuracy of a calculation. The higher the basis size the more accurate the result of the calculation, however the longer the time it takes. It can be seen from Table 8 that a relaxation with tier 3 basis takes more than ten times the time with minimum basis.

Table 9 shows an important aspect in geometry optimization. Although increasing the basis size is expected to provide better result, there was no gain in employing the maximum basis - the geometry and total energy were unchanged.

The version of FHI-aims used in this work support ten different LDA functional. The result of the total energy calculation using the optimized control.in file and the most stable geometry is given in Table 14. The smallest ground state total energy of $-13531.5152006310 \mathrm{eV}$ is predicted by the Rpbe function, while the highest of $-13416.0917537102 \mathrm{eV}$ is predicted by the Pz-Ida.

The calculation of vibrational frequencies involves all necessary DFT calculation, calling a routine to set up and diagnolize the Hessinan matrices, and the generation of the output stream. In the DFT calculations, six relxation steps are made for each atom. Undoutbly, calculation of the vibrational frequencies is tedious and time consuming. Therefore, in order to minimize the time the LDA exchande-correlation function was set to Vwn, the one which requires the shortest time.

A total of 51 vibrational modes were obtained, however the first three are unstable. The magnitudes of the frequencies of the first six modes shows that the input geometry is indeed a local minimum and not a saddle point. The intensity of some of these modes shows that $\alpha$-Benzopyrone can be a suitable sensitizer.

\section{Conclusion}

The findings of this work shows that $\alpha$-Benzopyrone can be a suitable sensitizer. It also demonstrated an 
error in the FHI-aims user manual on the SCFC stopping criteria.

\section{References}

[1] Aoife, L., \& Richard, O. (2004). Studies on coumarins and coumarin-releted compounds to determine their therapeutic role in the treatment of cancer. Current Pharmaceutical Design, 10, 3797-3811.

[2] Jain, P. K., \& Himanshu, J. (2012). Coumarin: Chemical and pharmacological profile. Journal of Applied Pharmaceutical Science, 2(6), 236-240.

[3] Rao, S. B. (2008). Chemistry of Coumarins, Technical White Paper. Indofine Chemical Company, Hillsborough.

[4] Grätzel, M. (2003). Sensitized solar cells. Journal of Photochemistry and Photobiology C; Photochemistry Reviews, 4, 145-153.

[5] Dye Sensitised Solar Cells. Retrieved from www.diss.fu-berlin.de

[6] O’Regan, B., \& Gratzel, M. (1991). A low-cost, high-efficiency solar cell based on dye-sensitized colloidal TiO2 films. Nature, 353, 737-740.

[7] Chiba, Y., Islam, A., Watanabe, Y., Komiya, R., Koide, N., \& Han, L. (2006). Dye-sensitized solar cells with conversion efficiency of 11.1\%. Jpn. J. Appl. Phys., 45, L638-L640.

[8] Ito, S., Murakami, T. N., Comte, P., Liska, P., Gratzel, C., Nazeeruddin, M. K., \& Gratzel, M. (2008). Fabrication of thin film dye sensitized solar cells with solar to electric power conversion efficiency over 10\%. Thin Solid Films, 516, 4613-4619.

[9] Toyoda, T., Sano, T., Nakajima, J., Doi, S., Fukumoto, S., Ito, A., Tohyama, T., Yoshida, M., Kanagawa, T., Motohiro, T., Shiga, T., Higuchi, K., Tanaka, H., Takeda, Y., Fukano, T., Katoh, N., Takeichi A., Takechi, K., \& Shiozawa, M. (2004). Outdoor performance of large scale DSC modules. J. Photochem. Photobiol. A, 164, 203-207.

[10] Berginc, M., Opara, K. U., Jankovec, M., \& Topiè, M. (2007). The effect of temperature on the performance of dye-sensitized solar cells based on a propyl-methyl-imidazolium iodide electrolyte. Sol. Energy Mater. Sol. Cells, 91, 821-828.

[11] Durr, M., Schmid, A., Obermaier, M., Rosselli, S., Yasuda, A., \& Nelles, G. (2005). Low-temperature fabrication of dye-sensitized solar cells by transfer of composite porous layers. Nat. Mater, 4, 607-611.

[12] Pichot, F. O., Pitts, J. R., \& Gregg, B. A. (2000). Low-temperature sintering of $\mathrm{TiO}_{2}$ colloids: Application to flexible dye-sensitized solar cells. Langmuir, 16, 5626-5630.

[13] Hara, K., Sato, T., Katoh, R., Furube, A., Ohga, Y., Shinpo, A., Suga, S., Sayama, K., Sugihara, H., \& Arakawa, H. (2003). Molecular design of coumarin dyes for efficient dye-sensitized solar cells. J. Phys. Chem. B, 107, 597.

[14] Faber, C., Duchemin, I., Deutsch, T., \& Blasé, X. (2012). Many-body green’s function study of coumarins for dye-sensitized solar cell. Phys. Rev. B, 86(15), 155-315.

[15] Hohenberg, P., \& Kohn, W. (1964). Inhomogeneous electron gas. Phys. Rev., 136, B864.

[16] Kohn, W., \& Sham, L. J. (1965). Self-consistent equations including exchange and correlation effects. Phys. Rev., 140, A1133.

[17] Engel, E. (2002). Orbital-dependent functional for the exchange-correlation energy. In Fiolhais, C., Nogueira, F., \& Marques, M. (Eds), A Primer in Density Functional Theory. Springer, Berlin.

[18] Barth, U. (2004). Basic density-functional theory - An overview. Physics Scripta, T109, 9-39.

[19] Blum, V., Gehrke, R., Hanke, F., Havu, P., Havu, V., Ren, X., Reuter, K., \& Scheffler, M. (2009). Ab initio molecular simulations with numeric atom-centered orbitals. Computer Physics Communications, 180, 2175-2196. 


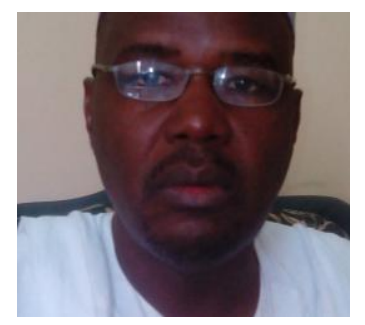

G. Babaji was born in Ningi, Bauchi State, Nigeria in 1959. Babaji's qualifications include the B.Sc in physics from Bayero University, Kano, Nigeria, 1984; the M.Sc in physics from Bayero Unversity, Kano, Nigeria, 1989; and the Ph.D in physics, University of Ibadan, Ibadan, Nigeria, 1998.

He joined the services of Bayero University, Kano in 1985 as a graduate assistant and rose to the rank of professor. In the past, he served as the head of Physics Department in Bayero University, Kano and in Umaru Musa Ya'radua University, Katsina. He is the author of the book Computational Physics Methods for Undergraduates (Ibadan, Nigeria: University Press PLC, 2010). His current research interests include molecular dynamics, and dye-sensitized solar cells.

Professor Babaji is the current president of the Nigerian Association of Mathematical Physics. He is a member of the Science Association of Nigeria, Nigerian Institute of Physics, and the Academic Staff Union of Universities. 